\title{
The End of Accretion: Transition Disks, Dissipation, and Links to Planet Formation
}

\author{
James Muzerolle ${ }^{1}$ \\ ${ }^{1}$ Space Telescope Science Institute, Baltimore, MD, 21218, USA \\ email: muzerol@stsci.edu
}

\begin{abstract}
I review recent studies of transitional protoplanetary disks, in which the planet forming regions are being cleared of material. The dust and accretion characteristics of these objects as a function of their environment and host star age and mass reveal important clues as to how disk structure is modified and ultimately destroyed. Clearing mechanisms such as grain growth, dynamical interactions with embedded planets or stellar companions, and photoevaporation are likely all involved, pointing to diverse disk evolutionary paths.
\end{abstract}

Keywords. accretion, accretion disks; circumstellar matter; planetary systems: protoplanetary disks; stars: pre-main-sequence

\section{Introduction}

Observational estimates of the lifetimes of circumstellar disks are essential for constraining planet formation theories. Studies focused on near-infrared emission probing dust in the innermost regions of disks have shown a clear decline in excess frequency with stellar age, indicating typical disk lifetimes of roughly $3 \mathrm{Myr}$, with a wide dispersion from 1-10 Myr. Meanwhile, measurements of mass accretion rates have provided complementary information on the gas content of disks, showing a general decline with stellar age consistent with expectations from models of viscous evolution. However, there is a large dispersion in accretion rates, as well as disk lifetimes, at any given stellar age that is not consistent with simple evolutionary models. The mechanisms for disk dispersal, including grain/planetesimal growth, giant planet formation, and photoevaporation, are still very much open to debate.

A key piece of the puzzle is provided by the small number of disks in transition. Such objects still harbor substantial amounts of primordial material but show signs of significant clearing in their inner regions. A handful of such transition disks with inner "holes" have been studied in some detail in recent years, particularly with the advent of Spitzer Space Telescope observations. Modeling of spectral energy distributions (SEDs) of transitional disks have inferred inner holes ranging from $\sim 3-24$ AU in size (e.g., Calvet et al. 2005). Follow-up interferometric observations of some of these objects have directly imaged these holes (e.g., Hughes et al. 2009; Brown et al. 2009).

\section{Transition disk statistics}

Relatively large numbers of transition disks are now being identified from Spitzer surveys of star forming regions and young stellar clusters. Below I list some of the important results that have been found to date on the properties of these objects.

- 3 types of transition disks: (1) classical transition disks, sources with small or zero dust excess at $\lambda \lesssim 10 \mu \mathrm{m}$ indicative of an optically thick outer disk with an AU-scale inner "hole" where the dust has been rendered optically thin or completely evacuated; (2) 
gapped or "pre-transitional" (Espaillat et al. 2007) disks, objects that exhibit a similar SED dip but more substantial short-wavelength excess indicative of an AU-scale disk gap bounded by optically thick inner and outer disk annuli; (3) "weak-excess", "evolved", or "homologously-depleted" disks, sources with small or zero short-wavelength excess and weak mid-infrared excess suggestive of a flat or optically thin disk.

- Accretion properties: A majority of type 1 and 2 objects show evidence for active accretion. This indicates that they still harbor substantial amounts of gas within their dust holes/gaps, and suggests that planetesimal or giant planet formation may be more likely in these cases. A much smaller fraction of type 3 objects show significant accretion activity, indicating either a lack of gas in the inner disk or very small accretion rates. When combined with the generally low disk masses inferred by Cieza et al. (2008), this suggests that photoevaporation may be an important mechanism for the type 3 transition.

- Trends with stellar age: The frequency of type 1 transition disks increases with stellar age $(\sim 1 \%$ of the total stellar population at $1 \mathrm{Myr}$ and $\sim 5-10 \%$ at $5 \mathrm{Myr}$; Muzerolle et al. 2009). The trend is even stronger when type 3 objects are included; these comprise a majority of the disks still present at 5-10 Myr. The frequencies suggest that the clearing duration may be age-dependent, and at least in the case of type 3 objects may be of order the total disk lifetime (e.g., Currie et al. 2009).

- Trends with stellar mass: There is an apparent deficit of type 1 transition disks around stars with masses $\mathrm{M} \lesssim 0.3 \mathrm{M}_{\odot}$ (Muzerolle et al. 2009). By contrast, the vast majority of type 3 transition disks are around M-type stars. The clearing mechanisms responsible for the different transition types thus apper to depend on the stellar mass. One possibility for the type 1 discrepancy is that giant planet formation is less common in disks around low mass stars. Conversely, the long lifetime of type 3 disks may be explained if photoevaporation is less efficient around low mass stars.

- Stellar multiplicity: High spatial resolution imaging has shown that at least one transition disk, the type 1 object CoKu Tau/4, is in fact circumbinary (Ireland \& Kraus (2008)). However, the recent survey of Kraus et al. (see Kraus, this volume) has failed to find stellar companions to any other Taurus stars with transition disks. The age and stellar mass trends are also inconsistent with binarity being a significant contributor.

The properties and trends of transition disks suggest the existence of multiple evolutionary pathways (Lada et al. 2006; Cieza et al. 2007). More than one process is likely involved with the clearing of disk material. Further detailed measurements of properties such as disk masses, mass accretion rates, and hole sizes are needed to better constrain which of these mechanisms may be at work in any given object (Najita et al. 2007).

\section{References}

Brown, J. M., Blake, G. A., Qi, C. et al. 2009, ApJ, 704, 496

Calvet, N. et al. 2005, ApJ, 630, L185

Cieza, L., Padgett, D. L., Stapelfeldt, K. R. et al. 2007, ApJ, 667, 308

Cieza, L. A., Swift, J. J., Mathews, G. S., \& Williams, J. P. 2008, ApJ, 686, L115

Currie, T., Lada, C. J., Plavchan, P., Robitaille, T. P., Irwin, J., \& Kenyon, S. J. 2009, ApJ, 698,1

Espaillat, C. et al. 2007, ApJ, 664, L111

Hughes, A. M. et al. 2009, ApJ, 698, 131

Ireland, M. J. \& Kraus, A. L. 2008, ApJ, 678, L59

Lada, C. J., Muench, A. A., Luhman, K. L. et al. 2006, AJ, 131, 1574

Muzerolle, J., Allen, L., Megeath, S. T. et al. 2009, ApJ, submitted

Najita, J., Strom, S. E., \& Muzerolle, J. 2007, MNRAS, 378, 369 